\title{
Influence of temperature inversions on air pollution in Krasnoyarsk
}

\author{
Alexander Dergunov ${ }^{1 *}$, Oleg Yakubailic ${ }^{2}$ \\ ${ }^{1}$ Federal Research Center Krasnoyarsk Science Center SB RAS, Krasnoyarsk, 660036, Russian \\ Federation \\ ${ }^{2}$ Institute of Computational Modelling SB RAS, Krasnoyarsk, 660036, Russian Federation
}

\begin{abstract}
The paper analyzes the meteorological conditions in Krasnoyarsk in the period from 2019 to 2020 . The relationship between the occurrence of temperature inversions in the surface layer of the atmosphere and air pollution by suspended solid particles $\left(\mathrm{PM}_{2.5}\right)$ is investigated. The paper uses a set of meteorological data of the NCEP GFS weather forecast model on air temperature on three isobaric surfaces $(1000,925$ and $850 \mathrm{mb})$, wind gusts and the height of the atmospheric boundary layer. The air monitoring system of the KSC SB RAS provides data on solid particle concentrations $\mathrm{PM}_{2.5}$ and wind speed. The relationship between the presence of temperature inversions in the lower layer of the atmosphere and periods of significant air pollution is shown, as well as the dependence of changes in wind speed and the height of the boundary layer of the atmosphere with changes in the average daily $\mathrm{PM}_{2.5}$ concentration. The possibility of using the meteorological data of the NCEP GFS model to study the surface layer of the atmosphere and the periods of its pollution, predicting the deterioration of the environmental situation in Krasnoyarsk, is demonstrated.
\end{abstract}

\section{Introduction}

Atmospheric pollution is one of the main problems of large cities in the world. As a result of the anthropogenic factors, a large amount of harmful substances that pollute the air of large metropolitan areas enter the atmosphere [1]. Studies show an association between increased concentrations of particulate matter (PM) in the air and the deterioration of human health [2]. During periods of unfavorable meteorological conditions (UMC), there is a sharp increase in the concentration of pollutants in the lower layer of the atmosphere. We are talking about industrial and automobile emissions, furnace heating, and so on [3]. One of the determining factors of UMC is the temperature inversion in the atmosphere. This is a layer of warm air that traps the vertical dispersion of harmful impurities [4].

There is a need to study atmospheric processes with the help of meteorological data sets to analyze periods leading to environmental degradation.

Sets of meteorological data can be obtained, for example, from ground-based environmental monitoring stations. The advantages of such weather information include a

* Corresponding author: alexdergunov@,icm.krasn.ru 
fairly high accuracy of measurements on the spot. However, the disadvantages of such data are that basic parameters limit such data sets, and the ground stations themselves can be located at a great distance from each other, which imposes certain restrictions on the spatial coverage of the problem being solved.

An alternative option is the weather data of various weather forecast models, for example, the Global Forecast System model. These sources provide many different layers of meteorological information on dozens of vertical levels, plotted on regular rectangular grids covering the entire Globe. The spatial resolution of such data can vary from 2.5 to 0.25 degrees and higher [5]. Developers try to improve this parameter regularly.

The purpose of this work is to analyze the influence of temperature inversions in the surface layer of the atmosphere on the formation of an unfavorable environmental situation in the city of Krasnoyarsk in 2019-2020 based on high-spatial-resolution meteorological data of the NCEP Global Forecast System weather forecast model. Identification of the relationship between the periods with the average daily concentrations of $\mathrm{PM}_{2.5}$ particulate matter suspended in the air, exceeding the average daily maximum permissible concentrations (MPC), the speed, wind gusts, and the height of the atmosphere's boundary layer.

The research area in this work is the city of Krasnoyarsk and part of the adjacent territories (Fig. 1).

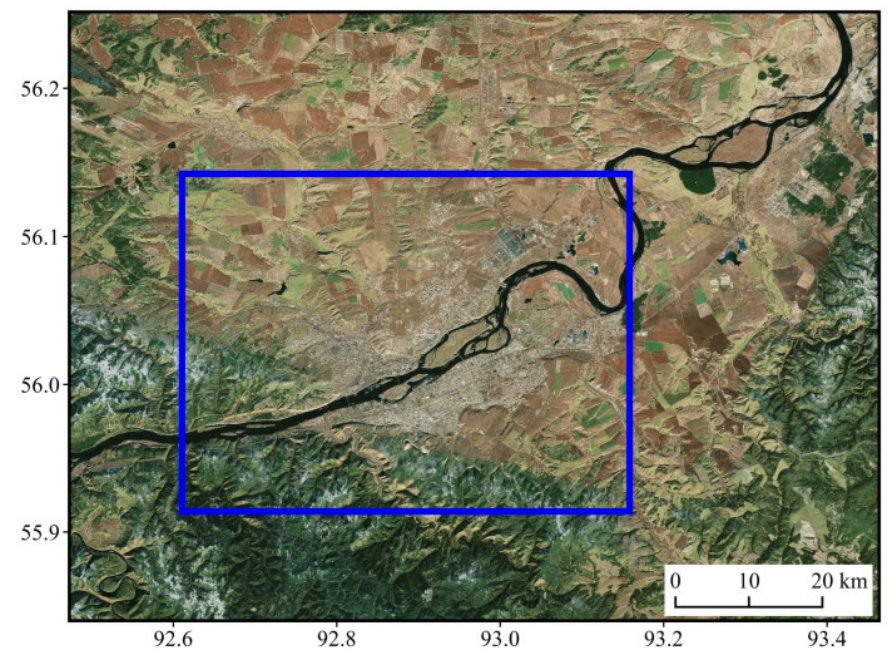

Fig. 1. The study area is the city of Krasnoyarsk.

12 periods of UMC were established in the city of Krasnoyarsk from 2019 to 2020 according to the Ministry of Ecology and Rational Nature Management data of the Krasnoyarsk Territory [6]. The periods of UMC are characterized by low wind speed and high particulate matter concentrations, significantly exceeding the average daily MPC equal to $0.035 \mathrm{mg} / \mathrm{m}^{3}$.

\section{Materials and methods}

The work uses meteorological information from the dataset of the Global Forecast System (GFS) model. This is a numerical weather forecasting system containing a global computer model and variational analysis performed by the US National Weather Service. This model combines four separate models: atmosphere, ocean, land/soil, and sea ice. Dozens of atmospheric and ground-soil variables are available in this data set, from temperature, wind, 
and precipitation, to soil moisture indicators and atmospheric ozone concentration. This is one of the most famous meteorological models in the world. Global data analysis and forecasting are carried out 4 times a day. The weather forecast is available up to 16 days in advance [5].

The accuracy of the GFS model is constantly improving. In particular, data with a horizontal resolution of 1 degree has been available since March 2004, with a resolution of 0.5 degrees since January 2007. Since January 2015, the horizontal resolution has been 0.25 degrees (about $25 \mathrm{~km}$ at the latitude of the city of Krasnoyarsk).

The GFS model data is presented in the grib2 format. This data format is standardized by the World Meteorological Organization (WMO) and is intended for storing historical and forecast weather data [7]. Each file contains more than 500 layers of various meteorological information on more than a hundred vertical levels.

This work used the actual analysis data on the air temperature at three vertical levels corresponding to three isobaric surfaces: 1000, 925, and $850 \mathrm{mb}$. Data on the height of the atmospheric boundary layer and information on wind gusts from January 1, 2019, to December 31, 2020, obtained from the GFS model data set, were also analyzed.

Inversion layers were detected by obtaining the difference between temperature data at Different vertical levels when the difference was negative. For example, with a negative difference between temperatures on isobaric surfaces of 1000 and $925 \mathrm{mb}$, the inversion was considered ground-level or elevated; if between 925 and $850 \mathrm{mb}$, it was elevated or highaltitude (inversion of the free atmosphere). If the surface elevated and high-altitude inversions were recorded simultaneously, this was considered a powerful inversion.

We also used data on the concentrations of $\mathrm{PM}_{2.5}$ in the air and wind speed according to ground-based monitoring stations obtained at the ICM SB RAS geoportal. Data on $\mathrm{PM}_{2.5}$ concentrations were averaged per day for all available observation posts in the city. Thus, the average daily values of the $\mathrm{PM}_{2.5}$ concentration for the entire city of Krasnoyarsk were obtained.

\section{Results and discussion}

Based on the analysis of the GFS data on air temperature at various vertical levels, a comparison was made of the number of days with temperature inversions and days when the average daily $\mathrm{PM}_{2.5}$ concentration exceeded the average daily MPC, by months for 2019 and 2020 (Fig. 2).
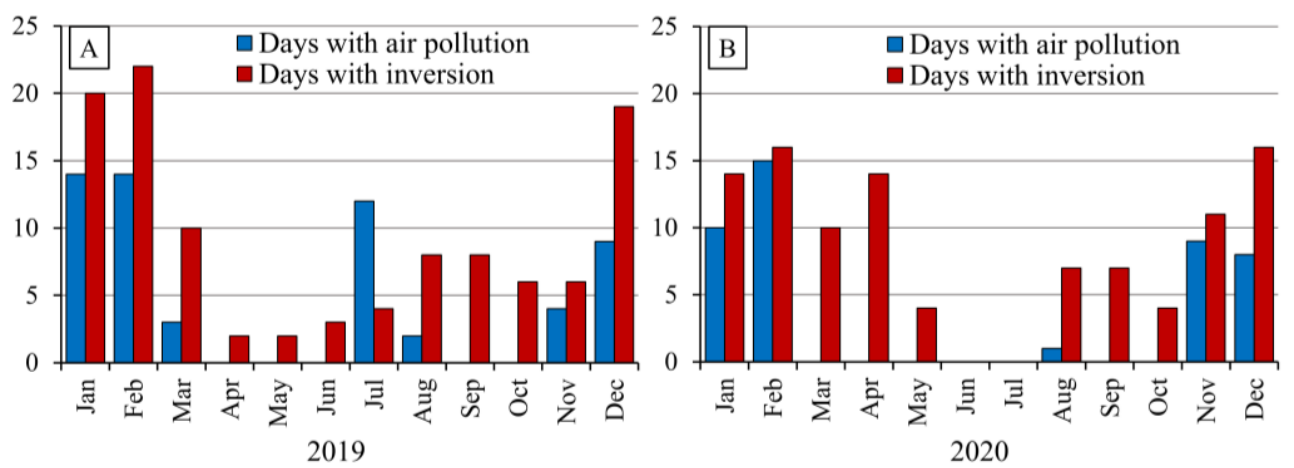

Fig. 2. Ecological situation in Krasnoyarsk by months in 2019 and 2020.

In April, May and November, the number of days with inversions is 19 days more in 2020 , in the rest of the months - on the contrary, with the exception of March, when both in 
2019 and in 2020 their number was 10 days. In 2019, there were 15 more days with "pollution" of the atmosphere than in 2020.

The total number of days with temperature inversions in 2019 was 110 , in $2020-103$ days. The total number of days with "pollution" of the atmosphere in 2019 was 58, in 2020 43 days.

Also, data analysis showed a stable relationship between changes in the average daily $\mathrm{PM}_{2.5}$ concentration and wind gusts according to the GFS model. As an example, the period from January to February 2020 is shown (Fig. 3). This period is characterized by the fact that out of 60 days within two months, 24 days were recorded serious air pollution with $\mathrm{PM}_{2.5}$ particulate matter.

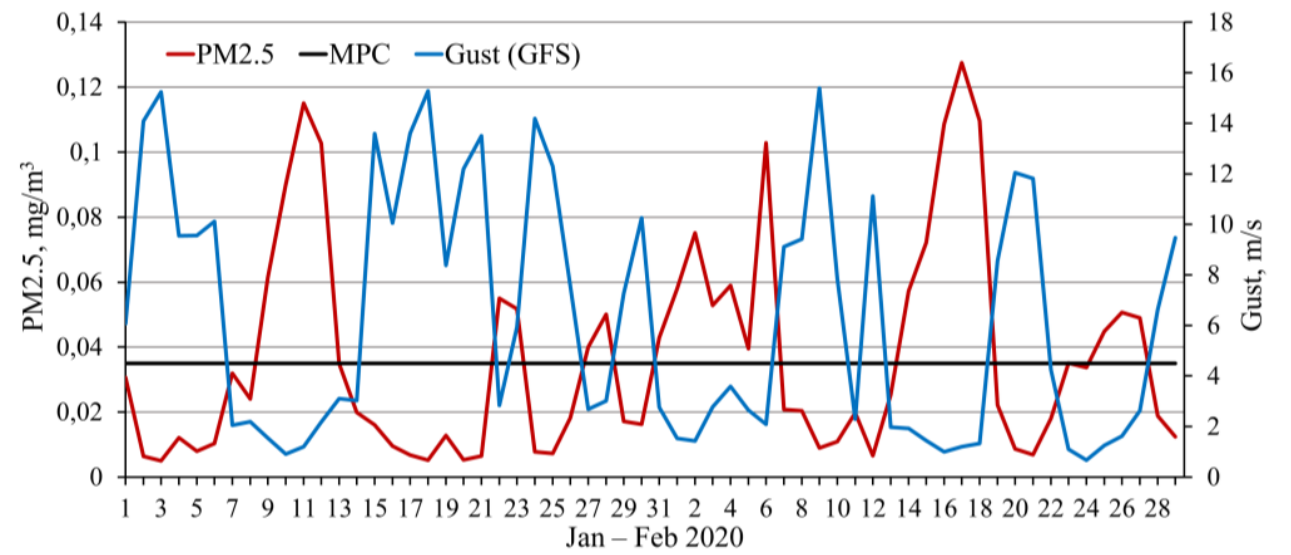

Fig. 3. Meteorological situation in Krasnoyarsk in January-February 2020. The red graph indicates the change in the average daily concentration of $\mathrm{PM}_{2.5}$, the blue graph - the change in the speed of wind gusts. The horizontal line indicates the average daily MPC, equal to $0.035 \mathrm{mg} / \mathrm{m}^{3}$.

Periods of increased $\mathrm{PM}_{2.5}$ concentration in January-February 2020: 09-13.01, 22-23.01, 27-28.01, 31.01, 01-06.02, 14-18.02 and 25-27.02. Temperature inversions were observed on these days, with the exception of January 9, February 5 and 26. It is shown that with an increase in wind speed, the level of pollution decreases and vice versa (Fig. 3).

Graphs showing the values of temperature inversions $(\Delta \mathrm{T})$ in the night and daytime for the period from January to February 2020 were constructed (Fig. 4).

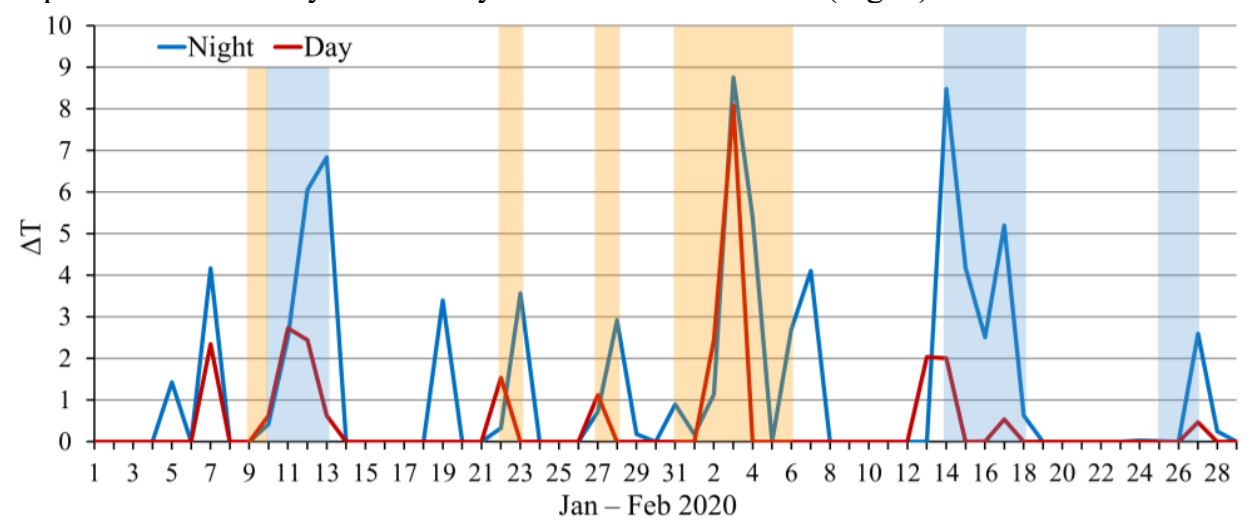

Fig. 4. Values of temperature inversions $(\Delta \mathrm{T})$ in January-February 2020. 
In Figure 4, the blue vertical stripes mark the official periods of UMC, and the orange vertical stripes indicate the periods with an increased average daily $\mathrm{PM}_{2.5}$ concentration identified as a result of data analysis.

Analysis of the data showed that, as a rule, the highest values of the value of temperature inversions are recorded during the marked periods of unfavorable environmental conditions in the city (Fig. 4). The prevailing type of inversion is ground-level or elevated. During the period under consideration, nighttime inversions appeared 52\% more often than daytime ones.

Additionally, the variation of the height of the planetary boundary layer of the atmosphere with the wind speed obtained from the data of the ground monitoring station was compared. The correlation coefficient was 0.79 . This is due to the fact that at high wind speed the stability of the atmosphere decreases, turbulence increases, which leads to an increase in the height of the boundary layer [8]. The correlation between the wind speed obtained from the ground monitoring station and the wind gusts from the GFS model is 0.82 .

\section{Conclusion}

As a result of the work done, the relationship between temperature inversion in the surface layer of the atmosphere and the periods of significant air pollution by $\mathrm{PM}_{2.5}$ solid particles in the city of Krasnoyarsk in 2019 and 2020 was shown.

The largest number of days with temperature inversions and increased air pollution is observed in the cold period (December, January, February). In 2019, inversions were registered in all 12 months of the year, and in 2020, there were no inversions only in June and July.

As a rule, the highest values of temperature inversions are recorded during periods of unfavorable environmental conditions in the city. The prevailing type of inversion is groundlevel or elevated. At night, temperature inversions appeared 52\% more often than during the day.

It is shown that with an increase in the wind speed and the height of the boundary layer, the average daily concentration of $\mathrm{PM}_{2.5}$ decreases.

The correlation between the wind speed obtained from the ground monitoring station and the wind gusts from the GFS model is 0.82 . Between the wind speed and the height of the boundary layer - 0.79 . Data on the height of the boundary layer can serve as an additional indicator when studying UMC periods in Krasnoyarsk.

The analysis of meteorological data of the GFS model contributes to solving problems related to the study of the lower layer of the atmosphere, its pollution and can play an important role in more accurately identifying periods of unfavorable meteorological conditions and forecasting them.

\section{References}

1. G. Zarubin, Y. Novikov, Hygiene of the city, Medicine, Moscow (1986).

2. Y.J. Kaufman, D. Tanre, O. Boucher. Nature. 419. №. 6903. P. 215-223. (2002).

3. A. Eremkin, I. Kvashnin, Y. Junkerov, Standardization of emissions of pollutants into the atmosphere, Publishing house of Ass. builds. universities, Moscow (2001).

4. L. Matveev, General meteorology course. Atm. Phys., Gidromet., Leningrad (1984).

5. The Global Forecast System (GFS) Documentation (2021). URL: https://is.gd/KJHKbK 
6. Unfavorable meteorological conditions in Krasnoyarsk. regional departmental information and analytical data system on the state of the environment of the Krasnoyarsk Territory (2021). URL: http://www.krasecology.ru/Nmu

7. NCEP WMO GRIB2 Documentation, Version 23.0.0 (2019). URL: https://www.nco.ncep.noaa.gov/pmb/docs/grib2/grib2_doc/

8. Y. Wang, H. Sayit, A. Mamtimin, J. Zhu, C. Zhou, W. Huo, F. Yang, X. Yang, J. Gao, X. Zhao. Atm. Res. 256 (2021). doi: 10.1016/j.atmosres.2021.105567 